\title{
To study the effect of low velocity impacts on composite material buy using Finite element analysis software LSDYNA
}

\author{
Mr.Nayeemuddin ${ }^{1}$, Mr. Abdul Nazeer ${ }^{2}$ \\ (Department of mechanical engineering, KBN college of engineering Gulbarga, Karnataka, India)
}

\begin{abstract}
During the life of a structure impact by foreign objects can be expected to occur during manufacturing, service and maintenance operations. An example of in service impact occurs during aircrafts takeoffs and landing. During the manufacturing process or during maintenance tools can be dropped on the structure, in this case the impact energy is small, laminated structures are more susceptible to impact damage often cannot be detected by visual inspection. This internal damage can severe reductions in strength and further reductions occur under the load. Therefore the effects of foreign object impact on composite structure must be understood and proper measures should be taken in the design process to account for expected events. Concerns about the effects of impact on the performance of composite structures have been a factor in limiting the use of composite materials. For these reasons the problem of impact has received considerable attention in the literature.

The process of impact damage initiation and growth, and identify the governing parameters are important for the formulation of numerical models for damage prediction, for designing impact resistant structures and for developing improved material systems. An important aspect of a model for predicting the impact dynamics is to accurately describe the behavior of the target under impact loading conditions. For some simple cases the structural loading can be modeled by a simple spring-mass system or by using the quasi-static behavior and considering the energy balance for a system. But in general more sophisticated beam, plate or shell theories are required to model the structure.
\end{abstract}

.Keywords: FEA, Low-velocity impact, LS DYNA, Modeling, Simulation

\section{Introduction}

Problem statement: To study the effect of low velocity impacts on composite material buy using Finite element analysis software LSDYNA

Problem Definition:- Low-velocity impact is defined as events which can be treated as quasi-static, the upper limit of which can vary from one to tens of $\mathrm{ms}^{-1}$ depending on the target stiffness, material properties and the impactor's mass and stiffness.

\section{Objective:}

- To review the literature on the low velocity impact on laminated composite.

- To study the relevant analytical and experimental models which are available in the literature.

- To conduct a simple analysis of rigid ball impact the steel plate and compare the obtained results with existing journal.

- To conduct the simple analysis on carbon fiber reinforced plastic (CFRP) plate by varying the mesh density of plate.

- To conduct the analysis on carbon fiber reinforced plastic (CFRP) plate by varying the mass and velocity of impactor.

- To conduct the analysis on the hybrid composite plate impacted by a rigid impactor.

- To predict the initiation and propagation of crack due to bending and delamination in laminated composite structure.

- Parametric studies involving impactor mass, velocity, laminate characteristics,boundary conditions etc. examining their influence on the impact response and on the extant of resulting damage.

- The obtained results are then compared with existing journal.

\section{Methodologies:}

A methodology describes different approaches to analyze the impact and the prediction of impact induced damage by means of the finite-element method can be categorized into the following steps: 
$>$ Determining the contact force between impacting mass and the plate element as a function of time. This contact force cannot be determined explicitly as it depends on the relative approach of the two contacting bodies.

$>$ Applying the contact force to find the transient dynamic response of the impacted structure as a function of position and time (displacements, strains, stresses, etc.) which depends on the mass and velocity of the impactor and the laminate characteristics (geometry, boundary conditions, ply arrangement, elastic properties etc.).

$>$ Refinement and pre-processing of the finite element model of a plate and sphere is carried out using LS DYNA.

D The finite element model impact simulation is carried out using LSDYNA as a solver.

$>$ Reviewing the results is done using LS-PrePOST as a post processor.

$>$ Comparing the obtained results with the existing journal.

.

\section{LS DYNA SOFTWARE}

Introduction to LS DYNA : LSDYNA is an explicit Finite Element program and is designed for transient dynamic analysis of highly nonlinear problems. In LS-DYNA the transient dynamic equilibrium equation is solved by the central difference method. Similar to ANSYS, LS-DYNA also uses Newton-Raphson method to solve the nonlinear problems including the contact and impact problems. For the contact problems, three distinct methods are used to handle such problems, includes the kinematic constraint method, the penalty method and the distributed parameter method.

\section{Advantages and Disadvantages of LS-Dyna}

The main advantages of LS-DYNA is that it is one of the most robust programs currently available, when simulating problems of nonlinear loading, which includes fluid structure interaction and impact problems. LS-DYNA has the capabilities to stand non linearity of the impact problem and possesses a wide database with different kind of material formulations. Also it has the ability to work with different kinematic descriptions and element types for dynamic analysis. The main disadvantage of this code is that it is not userfriendly and used a large number of variables and parameters to build a model.

LS-DYNA has a variety of element types, which include four-node tetrahedron and eight node solid elements, beam elements, three-node and four-node shell elements, eight-node solid shell elements, truss elements, membrane elements, discrete elements and rigid bodies. A variety of element formulations are also available for each element type. The most advantageous capability of LS-DYNA over other explicit finite element codes is its included contact algorithm. Several types of contact interfaces can be defined in LS-DYNA including surface to surface, nodes to surface, nodes tied to surface, and surface tied to surface contacts etc.

To solve the impact problems by LS-DYNA, the required input data can be classified into the following three categories:

a. Material properties: identified the material properties for the impactor and target object, can be a rigid body, isotropic material or composite material.

b. Initial and boundary conditions: specified the initial conditions for the impactor such as impact velocity, acceleration; boundary conditions for the target object such as clamped or simple support.

c. Contact conditions: defined the type of contact and friction coefficient, there are 18 different types of contact can be chosen to accurately represent the physical model, among them surface to surface (STS) and node to surface (NTS) is the most common use.

\section{Low velocity impact of a rigid impactor on steel plate}

To verify the accuracy of LS-DYNA, a steel plate impacted by a rigid ball. Here the contact force is calculated for the different mesh density keeping the same dimensions of the plate and impactor. The obtained results are compared with literature result.

\section{Geometric modeling of steel plate and rigid impactor}




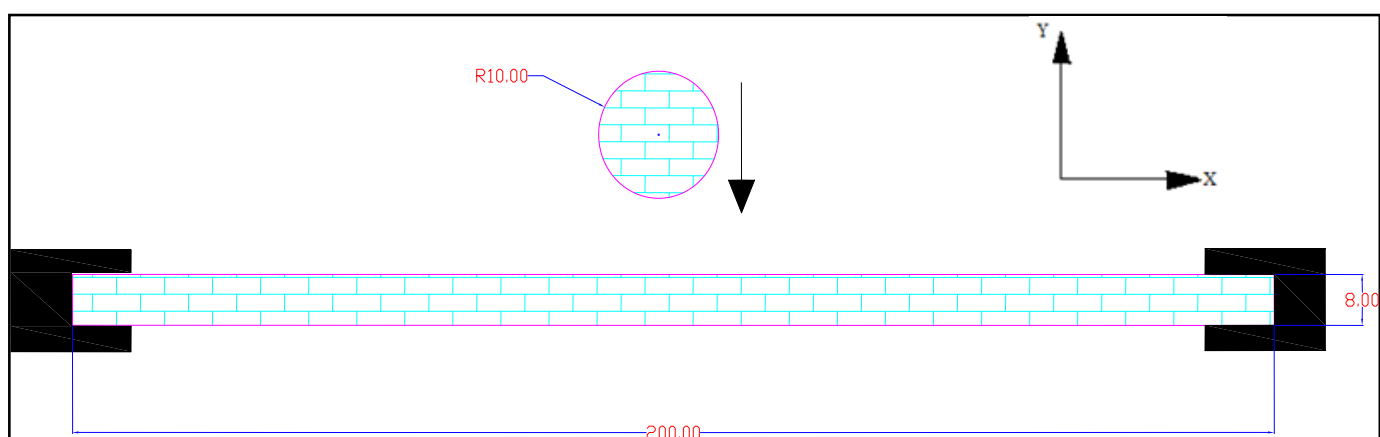

Figure 4.1: Geometric model of rigid impactor and steel plate

Figure 4.1 shows the geometric modeling of a rigid impactor and steel plate where the mesh density of the plate and sphere is varied from a corase mesh to a fine mesh with a clamped boundary condition the mesh density is varied in order to calculate the effect of mesh density on contact force.

\begin{tabular}{|c|c|}
\hline Parameters & Units \\
\hline Length unit & Millimeter $(\mathrm{mm})$ \\
\hline Time unit & Millisecond(msec) \\
\hline Mass unit & Kilogram $(\mathrm{Kg})$ \\
\hline Force unit & Kilo Newton(kN) \\
\hline Young's Modulus & Giga pascal $(\mathrm{Gpa})$ \\
\hline Density & $\mathrm{Kg} / \mathrm{mm}^{3}$ \\
\hline Velocity & $\mathrm{mm} / \mathrm{msec}$ \\
\hline
\end{tabular}

Table 4.1: Parameters and units

Table 4.2: Material Properties of Steel plate and Rigid Sphere

\begin{tabular}{|c|c|c|}
\hline Material properties for plate & Symbol (Unit) & Value \\
\hline Young's modulus & $\mathrm{E}(\mathrm{GPa})$ & 200 \\
\hline Poisson's ratio & $\gamma$ & 0.3 \\
\hline Density & $\rho\left(\mathrm{kg} / \mathrm{mm}^{3}\right)$ & $7.83 e^{-6}$ \\
\hline Yield stress & $\mathrm{f}_{\mathrm{y}}(\mathrm{GPa})$ & 0.2 \\
\hline & & \\
\hline Material properties for Sphere & Symbol $($ Unit $)$ & Value \\
\hline Young's modulus & $\mathrm{E}(\mathrm{GPa})$ & 207 \\
\hline Poisson's ratio & $\gamma$ & 0.3 \\
\hline Density & $\rho\left(\mathrm{kg} / \mathrm{mm}^{3}\right)$ & $7.85 e^{-6}$ \\
\hline Radius & $\mathrm{R}(\mathrm{mm})$ & 10 \\
\hline Velocity & $\mathrm{V}(\mathrm{mm} / \mathrm{ms})$ & 1 \\
\hline
\end{tabular}

\section{Assigning material in Ls-dyna}

The modeling meshing of composite plate is done in the Hypermesh 9.0 version preprocessor. The composite plate is modeled using Belytschko-Tsay shell elements there are wide range of material model in Ls-dyna the material model selected for assigning to plate is MAT 22 composite damage. The rigid sphere is modeled using Hughes-Liu solid elements material model assigned is MAT_20 MAT_RIGID the elements chosen are solid element. SMA wires are modeled using MAT_30 SHAPE_MEMORY.

The orientation and the through thickness location of the shape memory alloy (SMA) wires was used in order to minimize the maximum transverse deflection of the hybrid composite plate during the low-velocity impact phenomena.

To ensure the accuracy of the present model, the contact force determined from LS-Dyna model is compared to existing literature shokuhfar[2].

\section{Initial and Boundary conditions}




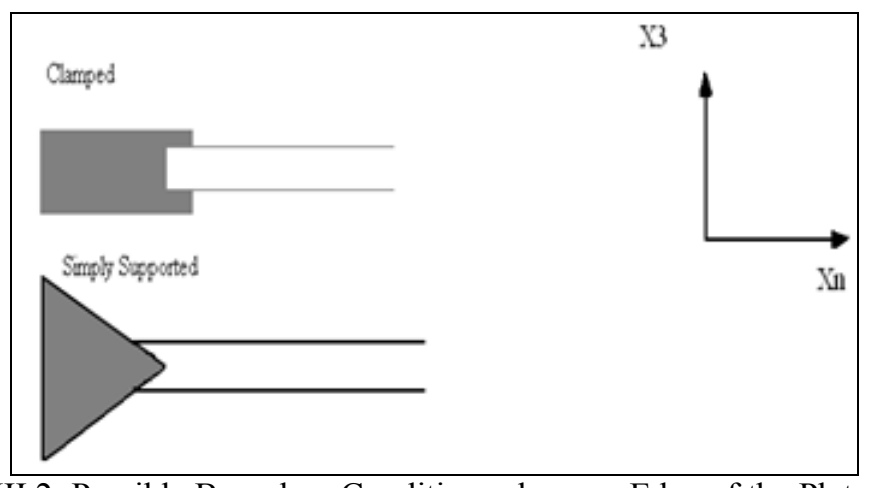

Figure III.2: Possible Boundary Conditions along an Edge of the Plate

Figure 6.11 shows the boundary conditions .initially (time $\leq 0$ ), the displacement and velocity at every point of the plate are zero.

Thus, the initial conditions are

$\mathrm{u}_{\mathrm{i}}=0 \quad \dot{u}_{\mathrm{i}}=\mathrm{O} \quad$ throughout the plate at $t \leq 0$

where $i=1,2,3$

At the start of the simulation (immediately time $>0$ ), the nodes that are in contact with the impactor are given the initial impact velocity, as well as the distributed mass of the projectile. Each edge of the plate may be clamped, simply supported or free. The boundary conditions corresponding to these types of support are

Clamped edge

$\mathrm{u}_{3}=0 \quad \mathrm{u}_{\mathrm{n}}=0$ on the surface of the edge

Simply supported edge

$\mathrm{U}_{3}=0 \quad \mathrm{u}_{\mathrm{n}}=0$ along the mid-line of the edge

\section{Defining laminated composite in LS DYNA}

By default, shells in LS-DYNA make the assumption of uniform transverse shear strain. This assumption is invalid for foam core composites or any stack up with lamina comprised of different materials with largely varying stiffness.

To correctly calculate the stiffness of sandwich composites (particularly foam cores) using shells, laminate theory must be invoked in LS-DYNA by any one of several means:

Use MAT_22, 54, or55

Use MAT_114, a plasticity model patterned after MAT_24.

Use MAT_116, a resultant layup-theory model.

\section{Modeling multi-layer composites}

There are several options for modeling layered composites with shell elements. The simplest and least general is to use the BETA option of SECTION_SHELL to define the material direction for each integration point through the element thickness. A userdefined integration rule should also be used to control the layer thicknesses. (IRID of SECTION_SHELL and INTEGRATION_SHELL).

If a composite is made up layers of different materials, a more general composite can be modeled by specifying a different part ID for each integration point (INTEGRATION_SHELL). Each part can refer to a different material model with the restriction that all materials must be of the same type. This method allows different material constants to be used in the different layers, but still does not allow completely general mixing of material types in a single shell element. In order to obtain the correct membrane and bending stiffness for the whole composite element, define a separate integration rule for each element in the composite with appropriate weights and through thickness locations.

\section{Assigning materials}

After creating and meshing the model the next step is to assign the material to the plate and sphere. LSDYNA is having a wide range of material and equation of state model each with a unique of history variables from the material library material model named MAT_PIECEWISE_LINEAR_PLASTICITY is used for the Sphere. A brief description of this particular material model is given below.

\section{MAT_PIECEWISE_LINEAR_PLASTICITY (Mat type 24)}

This is an Elasto-plastic material with an arbitrary stress versus strain curve and arbitrary strain rate dependency. The stress-strain relation for this material is defined with eight stress-vs.-strain points. With these material models, failure based on a plastic strain or a minimum time step size can be defined. In the FE model, this material is primarily used for the Sphere. 
MAT_COMPOSITE_DAMAGE (Mat type22)

An orthotropic material with optional brittle failure for composite can be defined following the suggestion of [Chang 1987a, 1987b].Three failure criteria are possible.

The Matrix Cracking Failure Criteria

$F_{\text {matrix }}=\left(\sigma / S_{2}\right)^{2}+\bar{\zeta}$

Where Failure is assumed Whenever Fmatrix $>1$.If Fmatrix $>1$ then the material constants $E_{2}, G_{12}, v_{1}$ and $v_{2}$ are set to zero.

The compression Failure Criteria is given as.

$\mathrm{F}_{\text {comp }}=\left(\sigma_{2} / 2 \mathrm{~S}_{12}\right)^{2}+\left[\left(\mathrm{C}_{2} / 2 \mathrm{~S}_{12}\right)^{2}-1\right] \sigma_{2} / \mathrm{C}_{2}+\bar{\zeta}$

Where Failure is assumed whenever $F_{\text {comp }}=>1$. If $F_{\text {comp }}>1$ then the material constants $E_{2}, v_{\square} \square$ and $v_{\square} \square$ are set to zero.

The Final Failure mode is due to fiber breakage

$F_{\text {fiber }}=\left(\sigma_{1} / S_{1}\right)^{2}+\bar{\zeta}$

Where Failure is assumed whenever $\mathrm{F}_{\text {fiber }}>1$. If $\mathrm{F}_{\text {fiber }}>1$ then the material constants $\mathrm{E}_{1}, \mathrm{E}_{2}, \mathrm{G}_{12}, \mathrm{v}_{\square} \square$ and $v_{\square} \square$ are set to zero.

By using the user defined integration rule,INTEGRATION_SHELL the constitutive constants can vary through the shell thickness. For all shell, except the DKT formulation, laminated shell theory can be activated to properly model the transverse shear deformation.

Material Properties and Element Formulation

After the material is assigned to the plate and sphere it is necessary to specify the properties of the material. SECTION_SHELL is used for composite and SECTION_SOLID is used for Steel sphere.

Shell element formulation: Element formulations are affiliated to the 'section properties of the respective elements type used. Following are the two types of shell element formulations used in the FE model.

Type 2(ELFORM 2/ EQ.2)- Belytschko-Tsay, used for composite plate.

Type 1(ELFORM1/EQ.1) - Hughes-Liu used for Steel sphere.

\section{Boundary Conditions}

Boundary conditions includes defining contacts, constraints etc. In this case contact between Steel ball and a composite plate are defined. Contact type used in LS-DYNA for impact is CONTACT_AUTOMATIC_NODES_TO_SURFACE. In LS-DYNA a contact is defined by identifying what locations are to be checked for potential penetration of a 'slave' node through a 'master' segment. As presently implemented, one surface of the interface is identified as a master surface and the other as slave. By using number of different algorithm, a search for penetration is carried out during the execution of solution In general, an input for the contact-impact algorithm, only the slave surface is defined and each node in the surface is checked each node in the surface is checked each time step to ensure that it does not penetrate through the surface. Internal logic identifies a master segment for each slave node and a slave segment for each master node and updates this information every time step as the slave and master nodes slide along their respective surfaces. Here the all the side of the plate is constrained to both translation and rotational moments i: e plate is clamped along all four sides.

\section{Defining Control time step}

The time step of an explicit analysis is determined as the minimum stable time steps in any deformable finite element in the mesh. For shell element the time step is given by the equation as shown below:

$$
\Delta \mathrm{t}_{\mathrm{e}}=\frac{L s}{C}
$$

Where, Ls is the characteristic length and

C is the sound speed

Time step for shell element can also be prevented by the equation as shown below

$$
\Delta \mathrm{t} \leq \frac{\Delta x}{C}
$$

Where, $\Delta_{\mathrm{x}}=$ Characteristic length

$\mathrm{E}=$ Young's modulus

$\rho=$ Density of the material

\section{Defining Database Binary Option}

The D3PLOT, D3PART, D3DRLF and the INTFOR files contain plotting information to plot data over the three dimensional geometry of the model. This database can be plotted with LS-PREPOST. The D3THDT file contains time history data for element subsets as well as global information.

Defining ASCII outputs 
ASCII output files (i.e. database files) are optional but necessary to obtain output files containing the results information. Below are some of the output flies which are specified:

GLSTAT: This file gives the global data. The output components for this file include kinetic energy, internal energy, total energy, ratio, stone-wall energy spring and damper energy, hourglass energy, sliding interface energy, external work, X, Y, Z- direction velocities.

MATSUM: This file outputs the individual material energies. The output components for this file includes Kinetic energy, Internal energy, hourglass energy, X,Y,Z-direction momentum, X,Y,Z- direction rigid body velocity of individual components with total kinetic energy, total internal energy, total hourglass energy.

NODOUT: This file gives the nodal point data. Output such as displacement in X, Y, Z directions, velocities in $\mathrm{X}, \mathrm{Y}, \mathrm{Z}$ direction, acceleration values in this direction with $\mathrm{X}, \mathrm{Y}, \mathrm{Z}$ direction rotation, rotational velocities and rotational acceleration can be obtained.

SECFORC: Cross sectional forces are obtained from the ASCII file SECFORC output such as forces in X, Y, Z direction, momentum in $\mathrm{X}, \mathrm{Y}, \mathrm{Z}$ direction, Cross sectional area after deformation, resultant force acting on the section specified can be obtained by this option.

RCFORC: This file gives the Resultant interface forces in $\mathrm{X}, \mathrm{Y}, \mathrm{Z}$ directions used for impact problems the resultant interface force is nothing but the contact force.

SLEOUT: This file gives the Sliding interface forces. This option is used for to find out the Slave energy and master energy.

\section{Low-Velocity Impact on steel plate with rigid impactor \\ IV. RESULT AND DISCUSSION}

The analysis is carried out on a steel plate. A rigid ball is impacted on a steel plate with different mesh density. The material properties of plate and Sphere is defined in Table 6.2 the plate with length $200 \mathrm{~mm}$, width $200 \mathrm{~mm}$ and thickness $8 \mathrm{~mm}$ is clamped along the four edges. Mesh 1 with 20 (length) x 20 (width) x 2 (height) $=800$ elements in plate and 32 elements in impactor. Mesh 2 with 40 (length) x 40 (width) x 2 (height) $=3200$ elements in plate and 32 elements in impactor. Mesh 3 with 80 (length) x 80 (width) x 2 (height) =12,800 elements in plate and 256 elements in impactor. LS-DYNA is capable of calculating the transient responses and provides the displacements and stresses distributions for both impactor and plate.

Once the model was made in Msc/Pattern and the key file was edited the model was run on a solver for solving the given loading and boundary constraints. The results were studied in post- solver LS-POST and compared with the results in the literature and good co relation was found between the simulations and results of SHIUH-CHUAN HER [7]

The impact analysis on a steel plate with the meshing of $20 \times 20 \times 2$

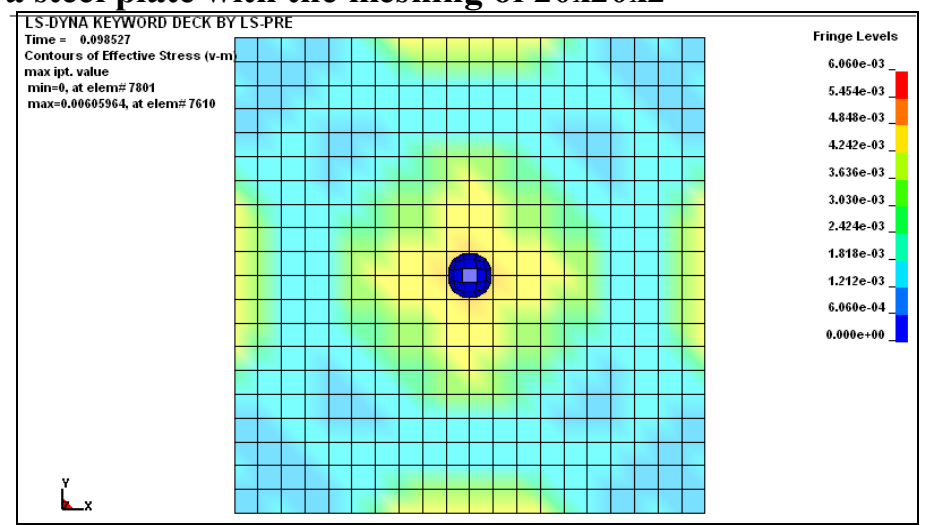

Figure 2.1: Stress plot for Steel plate at time $1 \mathrm{~ms}$ with $(20 \times 20 \times 2$,) mesh size and impactor velocity $1 \mathrm{~mm} / \mathrm{ms}$ 


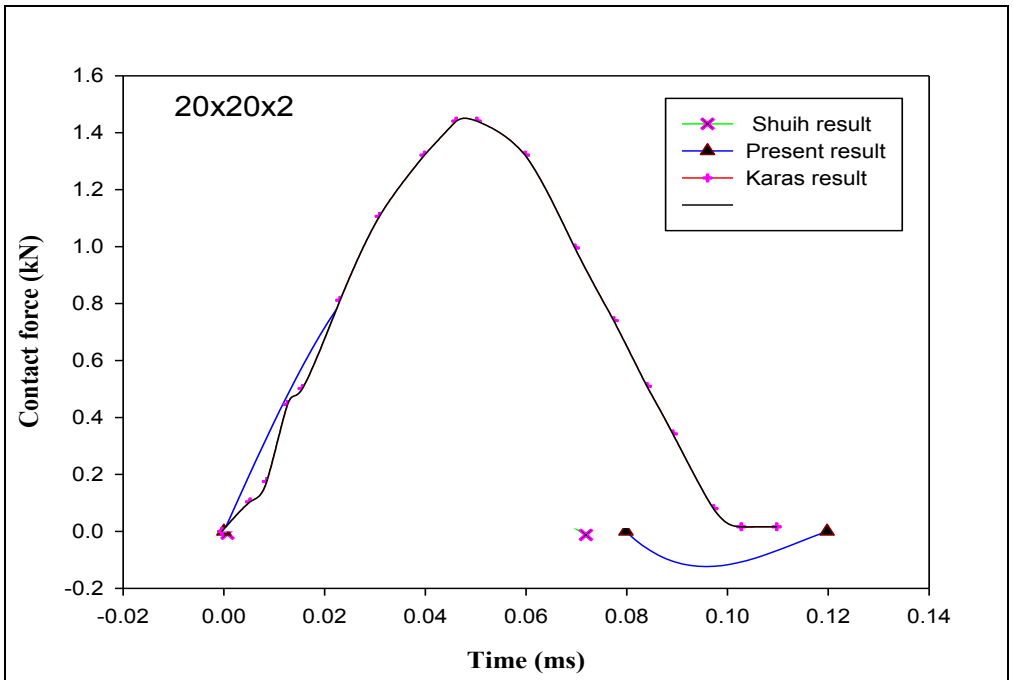

Figure 2.2: Comparison of contact force of present result and karas and shiuh[7]

Figure2.1 illustrate the damage caused to the Steel plate due to impactor with velocity $1 \mathrm{~m} / \mathrm{s}$ the region in red shows maximum stress condition. As the impact analysis is carried on different mesh density first we consider the coarse mesh to plate and sphere as $20 \times 20 \times 2=800$ elements and 32 elements in sphere. Figure 7.2 shows the comparison of contact force vs time between the present result and the result obtained from Shuih and karas as it can been seen that there is the difference in contact force obtained from Fem and Experimental result obtained from Karas.

\section{The impact analysis on a steel plate with the meshing of 40x40x2}

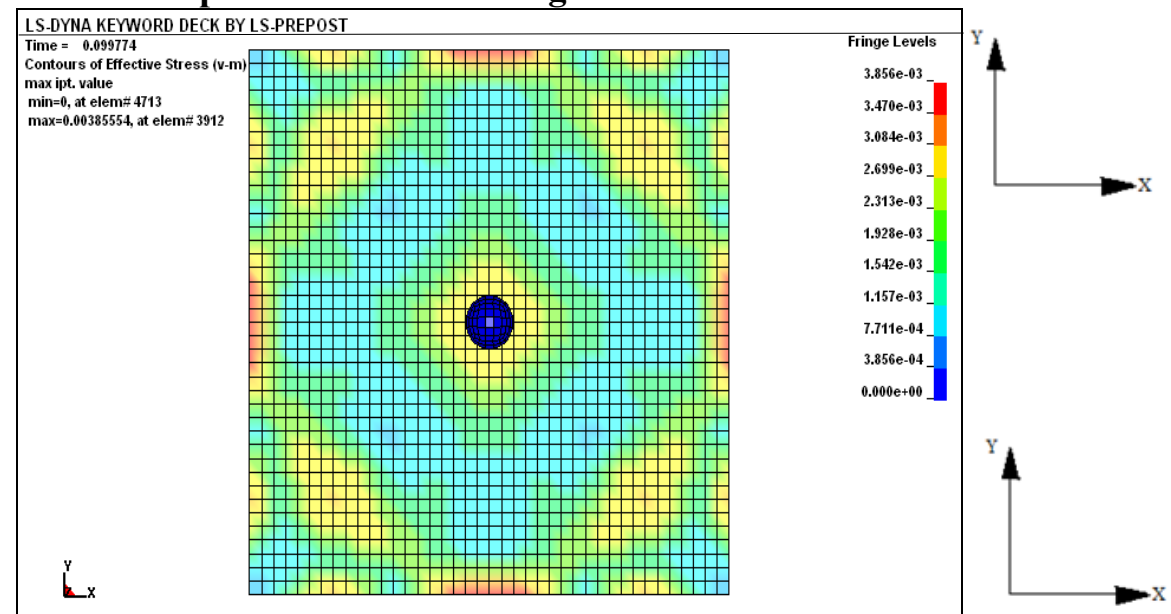

Figure 3.1: Stress plot for Steel plate at time $1 \mathrm{~ms}$ with $(40 \times 40 \times 2)$ mesh size and impactor velocity $1 \mathrm{~mm} / \mathrm{ms}$

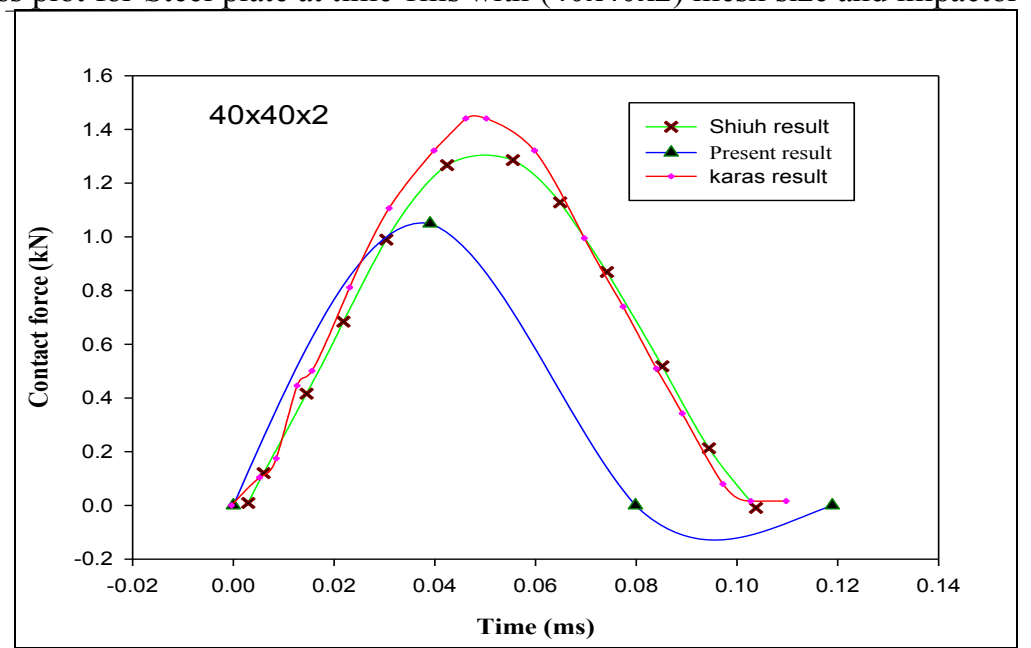

Figure 3.2: Comparison of contact force of present result and karas and shiuh[7] 
Figure 3.2 illustrate the damage caused to the steel plate due to impactor with velocity. $1 \mathrm{~m} / \mathrm{s}$ the region in red shows maximum stress condition as it can be seen from the figure 7.3 the maximum stress in red region is not below the impactor but it is away from the impact point. figure 7.4 shows the comparison between the result obtained from FEM and the Experimental result obtained by Karas there is a slight variation in the contact force. So it is seen that as the mesh density of plate is refined the contact force is increased and it is nearer to the result obtained by the Karas and Shuih.

\section{The impact analysis on a steel plate with the meshing of 80x80x2}

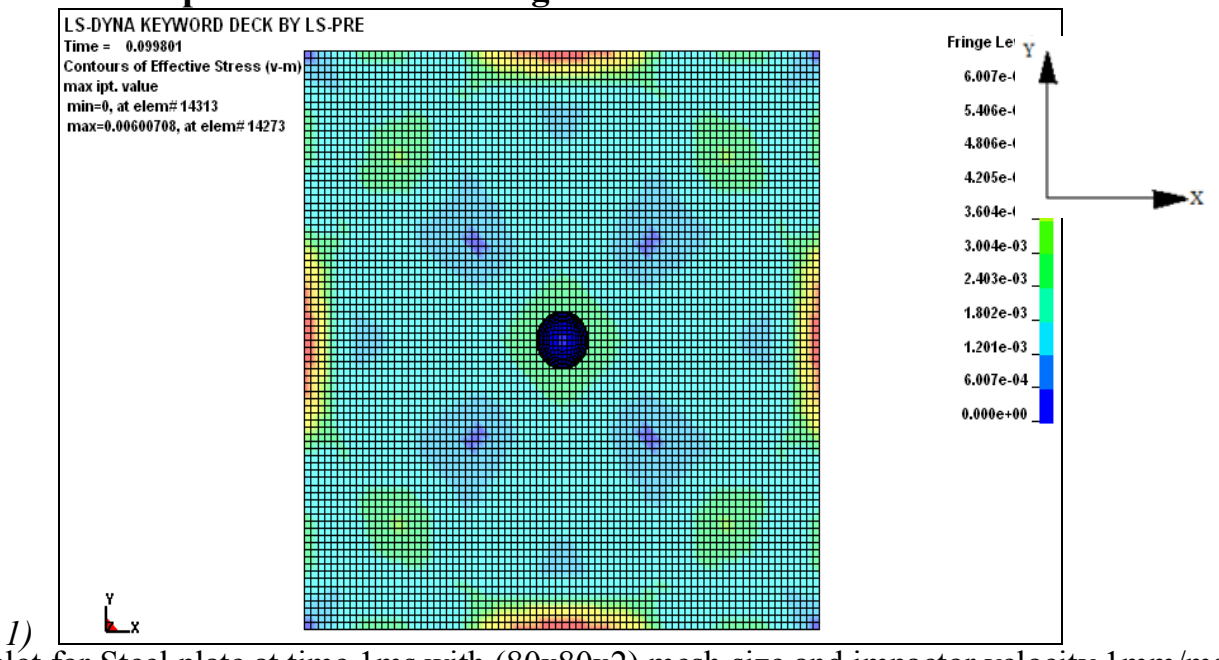

Figure 4.1: Stress plot for Steel plate at time $1 \mathrm{~ms}$ with $(80 \times 80 \times 2)$ mesh size and impactor velocity $1 \mathrm{~mm} / \mathrm{ms}$

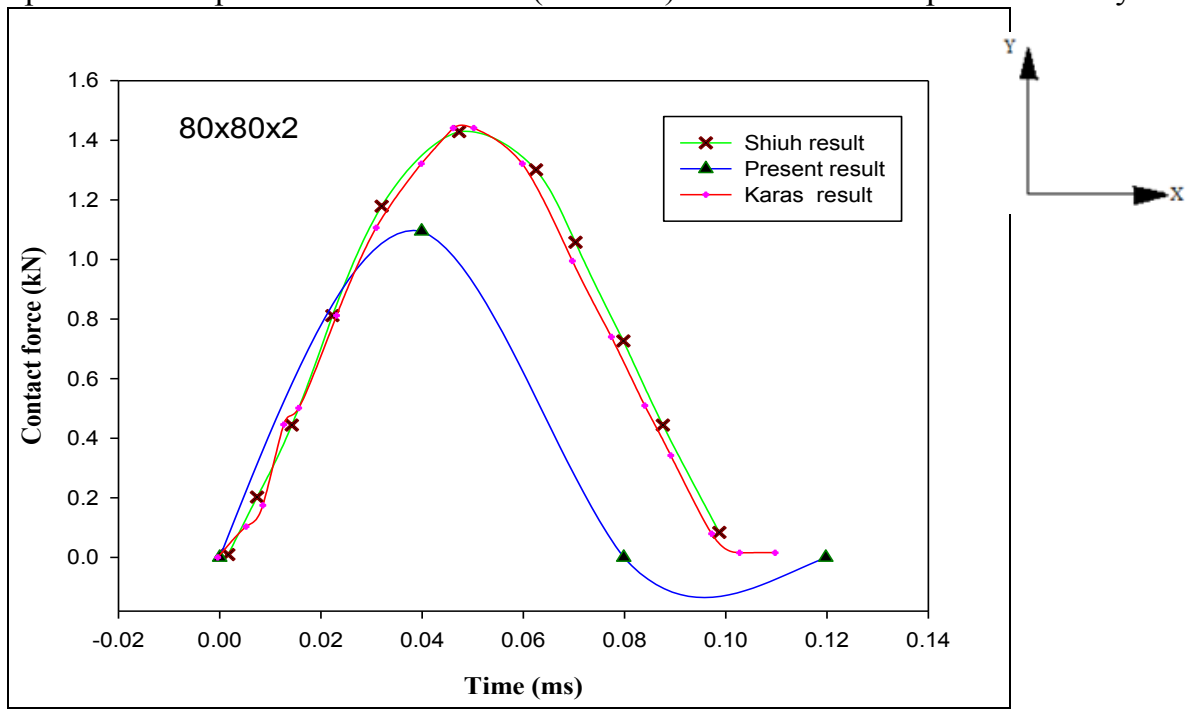

Figure 4.2: Comparison of contact force of present result and karas and shiuh[7]

Figure 4.1 illustrate the damage caused to the Steel plate due to impactor with velocity $1 \mathrm{~m} / \mathrm{s}$ the region in red shows maximum stress condition. Figure7.6 shows the comparison of contact force of present result and Karas and shuih and the result obtained by FEM is matching well with the literature [7]. So it can concluded the meshing of plate and sphere play very important role in analyzing the impact problem

Table 4.1: Comparison of FEM and Experimental Peak force

\begin{tabular}{|c|c|c|}
\hline Mesh Size & $\begin{array}{c}\text { FEM Simulation } \\
\text { Peak Impact Load }(\mathrm{kN})\end{array}$ & $\begin{array}{c}\text { Experimental Simulation } \\
\text { Peak Impact Load }(\mathrm{kN})\end{array}$ \\
\hline $20 \times 20 \times 2$ & 1.004 & 1.41 \\
\hline $40 \times 40 \times 2$ & 1.05 & 1.41 \\
\hline $80 \times 80 \times 2$ & 1.1 & 1.41 \\
\hline
\end{tabular}

\section{CONCLUSION}

A numerical analysis procedure for simulating the impact response of laminated composite Plates using finite elements codes has been presented. The present formulation has been used to predict the transient response of laminated composite plates and the obtained results have been successfully compared with existing 
experimental data and other theoretical solutions in the literature. In comparison with experimental measurements of the contact force histories for different impact conditions, the current predictions appear to be found fair agreement.

Firstly the analysis is carried out on a steel plate impacted by rigid impactor for different mesh density of plate and impactor. So it has been found that as mesh density increses the accuracy also increased. As shown from the figures2.2,3.2, and 4.2 the numerical results obtained by LS-DYNA can converge to the analytical solution Karas, upon the mesh being refined.

\section{REFERENCES}

[1]. IK-HYEON CHOI, "LOW-VELOCITY IMPACT ANALYSIS OF COMPOSITE LAMINATES UNDER INITIAL IN-PLANE LOAD” COMPOSITE STRUCTURES 86 (2008) 251-257

[2]. Shokuhfar.A, "Analysis and optimization of smart hybrid composite plates subjected to low-velocity impact using the response surface methodology (RSM)" Thin-Walled Structures 46 (2008) 1204- 1212

[3]. Tiebreak .R.a, Bachene.M b, Rechak S.c, Necib.B "Damage prediction in composite plates subjected to low velocity impact" Composite Structures 83 (2008) 73-82

[4]. SADIGHI. M, H. POURIAYEVALI, SAADATI.M "Response of fully backed sandwich beams to low velocity transverse impact" World Academy of Science, Engineering and Technology 362007

[5]. Hosur.M.V, "Studies on the low-velocity impact resip/;ponse of woven hybrid composites" Composite Structures 67 (2005) 253262

[6]. Ik Hyeon Choi, "Low-velocity impact analysis of composite laminates using linearized contact law" Composite Structures 66 (2004) 125-132

[7]. Shiuh-Chuan Her, "The finite element analysis of composite laminates and shell structures subjected to low velocity impact" Composite Structures 66 (2004) 277-285

[8]. Giovanni Belingardi, "Low velocity impact tests of laminate glass-fiber-epoxy matrix composite material plates" International Journal of Impact Engineering 27 (2002) 213-229

[9]. Krishnamurthy.K.S, "A parametric study of the impact response and damage of laminated cylindrical composite shells" Composites Science and Technology 61 (2001) 1655-1669

[10]. Parhi.P.K, Bhattacharyya.S.K and Shina.P.K:"Failure analysis of multiple delaminated composite plates due to bending and impact" Bull. Mater. Sci., Vol. 24, No. 2, April 2001, pp. 143-149

[11]. Mili.F, Necib.B, "Impact behaviour of cross-ply laminated composite plates under low velocities" composite structure 51 (2001) 237-244

[12]. Wade c. jackson and C.C. Poe.jr "The use of impact force as a scale parameter for the impact response of composite laminates" Nasa Technical Memorandum 104189 Avscom Technical Report 92-B-001

[13]. Silva M.A.G. and Cismaşiu.C Centro de Investigação em Estruturas e Construção - UNIC, Faculdade de Ciências e Tecnologia,sUniversidade Nova de Lisboa, Quinta da Torre, 2829-516 Caparica, Portugal.

[14]. Sheng-Tsong Chiu, "Low velocity impact behavior of prestressed composite laminates" Materials Chemistry and Physics 47 (1997) 268-272

[15]. Vaziri.R, Quan.X and Olson.M.D “Impact analysis of laminated composite plates and shells by super finite elements Int. J. Impact Enon9 Vol. 18, Nos 7 8, pp. 765-782, 1996

[16]. Jeffery.S.N, Paine and Craig A. Rogers. Centre for intelligent material system and structure virginia Tech Blacksburg, Virginia 24061-0261 vol-5 july 1994

[17]. Delfosse, D, Vaziri, R, Pierson M.O, and Poursartip, A., Analysis of the non-penetrating impact behavior of CFRP laminates. Proceedings of the 9th International Conference on Composite Materials, Madrid, Spain, July 1993, pp. 366-373

[18]. Liu.G.R and Quek.S.S "The finite element method A practicle course"

[19]. LS-DYNA KEYWORD USER'S MANUAL”, Version-971, Livermore Software Technology Corporation,2006

[20]. http://www.lsdyna.com 